Original Article

\title{
MACULAR OCT OUTCOMES OF PHACOEMULSIFICATION CATARACT SURGERY IN DIABETIC VERSUS NON DIABETIC PATIENTS
}

\author{
Ahmed, A. ${ }^{(*)}$, Abd El-Latif, I., Abozaid, M., Hafez, M. \\ Ophthalmology dept., Faculty of Medicine, Sohag Univ., Sohag, Egypt \\ *E-mail: alaamahmoud13102011@gmail.com
}

Received 12/2/2021

Accepted 22/5/2021

\begin{abstract}
Background: Cataract surgery with phacoemulsification is one of the most common surgical procedures performed today .Compared to people without diabetes, diabetic patients have been shown to have an elevated risk of developing cataracts. Cataract surgery in diabetics is indicated not only to improve visual acuity, but also to allow assessment and treatment of fundus changes. Even in the absence of diabetic retinopathy, diabetes mellitus has been found to have about twice the occurrence rate of pseudophakic CME. Objectives: This study was done to document any subsequent post-phacoemulsification macular edema (PPME) as postoperative sequelae in the diabetic versus non-diabetic nuclear senile cataract patients. Methods: This study was conducted on patients complained from visually significant immature senile cataract in ophthalmology department at Sohag university hospital between January 2018 and December 2020.Corrected distance visual acuity, Keratomerty, refraction, slit-lamp examination, macular optical coherence tomography (OCT) and biometry. Phacoemulsification was performed to all patients by stop and shop technique. The macular OCT changes were documented preoperatively and at postoperative month 1, 3, 6. Results: 64 eyes of 64 patients with visually significant senile cataract were included 32eye of them were diabetics, (33 males, and 31 females), our study documented four cases of the entire 64 eyes (6\%) complicated with PPME in groups $A$ and $B$ at the postoperative month 1 . Both $A$ and $B$ groups exhibited the same incidence of postoperative macular edema i.e. two (6\%) cases in each group. Conclusion: Our outcomes confirmed that there was a similar probability of PPME occurrence in both non-diabetic and diabetic patients as this probability was mostly related to the surgical procedure itself. Meanwhile, the non-diabetic PPME exhibited rapid and complete recovery within one month in comparison to the diabetic PPME that exhibited gradual and incomplete recovery during the postoperative six months follow-up period.
\end{abstract}

Keywords: Macular OCT, Cataract surgery, Phacoemulsification, Diabetes

\section{Introduction}

Cataract surgery with phacoemulsification is one of the most common surgical procedures performed today. Compared to people without diabetes, diabetic patients have been shown to have an elevated risk of developing cataracts [1,2]. Cataract surgery in diabetics is indicated not only to improve visual acuity, but also to allow assessment and treatment of fundus changes [3]. Good 
vision after Phacoemulsification cataract surgery requires healthy macula [4]. In diabetic patients, cystoid macular edema (CME) is more likely after cataract extraction. This may be partially due to increased blood-retinal barrier (BRB) breakdown or increased inflammation observed in diabetic patients following cataract extraction [5,6]. Even in the absence of diabetic retinopathy, diabetes

\section{Material and Methods}

\subsection{Study design}

This study gained the approval of the Institutional Review Board (IRB) in Faculty of Medicine, Sohag Univ., Egypt. Our study was designed as a comparative, prospective, non- randomized controlled interventional study. In addition, this study was registered at The Pan African Clinical

\subsection{Participants}

This study included 64 eyes of 64 patients with visually significant senile cataract. The study eyes were divided into two groups; group A (control/nondiabetic group) included 32 eyes of 32 patients who are non-diabetic and suffering from visual significant cataract, while group B (experimental /diabetic group) included 32 eyes of 32 patients

\subsection{Selection criteria}

The eligibility criteria of the study participants in group $\mathrm{A}$ as inclusion criteria were as follows: senile nuclear cataract (nuclear grades NI and NII according to the Lens Opacities Classification System III [9] non-diabetic, normal fundus examinations and within normal intraocular pressure (IOP) measurement. Our inclusion criteria in group B were as follows: senile nuclear cataract (nuclear grades NI and NII according to LOCUS III), 50 to 70 years old, well-documented diabetics with type II DM, DM $\geq 5$ years, with well-controlled glycemic value (glycosylated hemoglobin $<7 \%$ ), maintained mellitus has been found to have about twice the occurrence rate of pseudophakic CME. There has also been a higher prevalence of CME post-cataract surgery in eyes with diabetic retinopathy [7]. Those Patients may complain of metamorphopsia, central scotoma and reduced contrast sensitivity. Clinical examination shows loss of the foveal depression and retinal thickening [8].

Trial Registry (PACTR201804003056259) and was adhered to the Tenants of Helsinki. All surgeries were performed by one surgeon (A.M.) in ophthalmology department at Sohag university hospital between January 2018 and December 2020.

who are diabetics, non-retinopathy diabetic and suffering from visual significant cataract. The nature of both diseases; senile cataract and diabetes mellitus, the treatment modality options, potential intraoperative and postoperative consequences were carefully explained to all patients, who signed the informed consent before surgical intervention.

on oral hypoglycemic drugs, and normal fundus examinations and within normal intraocular pressure (IOP) On the other hand, our exclusion criteria included Patients with corneal pathology or low endothelium endothelial cell count $(<2000$ cell/mm), poor pupillary dilatation, pseudoexfoliation Syndrome, high IOP, glaucomatous optic neuropathy, previous history of ocular trauma or uveitis, previous eye surgery, current or previous macular edema, maculopathy or retinopathy, cataract density interfered with pre-operative ocular coherent tomography (OCT) imaging. 


\subsection{Interventions}

The first device used in our study macular OCT (OPTOVUE Wide field Enface OCT, Biotechnology company, Fremont, California, USA) that was used to obtain macular volume; foveal, parafoveal (including both superior and inferior hemisphere) and perifoveal (including both superior and inferior hemisphere) thicknesses), The second device was the phacomachine (INFINITI ${ }^{\circledR}$ OZIL continuous with IP software, Alcon, Forth Worth, Texas, USA. The first step was to instill topical anesthetics Benoxinate Hydrochloride $0.4 \%$ (Benox, Pharmaceutical Industries Company, E.I.P.I.CO, Tenth of Ramadan City, Egypt) into the patient eye. Then, to obtain adequate pupillary dilation before cataract surgery; the topical mydriatic eye drops cyclopentolate hydrochloride $50 \mathrm{mg}$ and phenylephrine hydrochloride 500mg (cyclophrine, Kahira pharm, Cairo, Egypt) was instilled into the patient eye every 10 minutes for 30 minutes. Thereafter, the patient eye underwent both retrobulbar and facial nerve blocks by injection of lidocaine $\mathrm{Hcl}$ 2\% (Xylocaine, AstraZeneca, Cambridge, UK). Povidoneiodine 10\% (Betadine, Antiseptic Solution, El-Nile $\mathrm{CO}$ for pharmaceuticals and chemical industries, Cairo, Egypt) was used for paintaing the skin of the eye lids, forehead and checks on the side of the requested eye. Furthermore, povidoneiodine 5\% eye drops were instilled into the eye to be followed by applying a disposable eye drape with fluid collection bag (Opsite plastic eye drap, Freedom Opthalmic Pate. Ltd., Tamil Nadu, India). The eyelids were maintained open by an eye-speculum. Two paracentesis incisions were performed by micro-vitreoretinal knife 20 Gauge (MVR 20G, $1.2 \mathrm{~mm}$ angled Blade Alcon laboratories Inc., Forth Worth, Texas, USA) followed by performing a clear corneal incision using Clear Cut $\mathrm{HP}^{2}$ Dual Bevel Slit knife (Keratome $2.2 \mathrm{~mm}$, Alcon laboratories, inc, Forth Worth, Texas, USA). We intended to perform a typical tri- planar wound to promote incision selfsealing. Air was injected into the anterior chamber (AC) via the paracentesis incision followed by injection of the trypan blue ophthalmic solution $0.6 \mathrm{mg} / \mathrm{ml}$ (Optiblu, Ophtechnics Unlimited, Haryana, India) into the $\mathrm{AC}$ in order to stain the anterior capsule. Thereafter, we injected compound sodium lactate solution (Ringer's lactate, I.V. infusion, Allmed middle east, $6^{\text {th }}$ of October, Egypt) into AC to wash the residual dye. Then, we injected a thick cohesive ophthalmic viscoelastic device (OVD); sodium hyaluronate ophthalmic solution 1.4\% (Optiflex, Moss Vision Inc. Ltd, Wembley, United Kingdom) into the AC. Regarding capsulorhexis, at first; we performed an angular tab in the anterior capsule. This tab was then pulled in a curvilinear motion and the continuous tear was then proceeded in a clockwise manner by grasping the tearing-edge with the capsulorhexis forceps to control the vector of the tear thus creating a regular circular opening. Hydrodissection was performed by injection of Ringer's lactate solution within a dispersive viscoelastic fluid; 2\% Hydroxypropyl Methylcellulose (Supreme viscoelastic surgical fluid, Rumex international Ltd, Yorkshire, UK) was then injected into anterior chamber. We planned to fixate the intraoperative phacomachine parameters. Regarding the phaco 1 parameters (Sculpting), we used the following parameters: torsional Amplitude was 100 linear, phaco power was 0 , vaccum was 95 linear, irrigation bottle height was $98 \mathrm{~cm} \mathrm{H}_{2} \mathrm{O}$ and aspiration flow rate was 23 linear. Regarding the phaco 2 parameters, we used the following parameters: torsional Amplitude was 95 linear, phaco power was 0 , vaccum was 400 linear, irrigation bottle height was $95 \mathrm{~cm} \mathrm{H}_{2} \mathrm{O}$, aspiration flow rate was 25 panel, dynamic Rise was -2 and Intelligent phaco was on. Regarding the epinucleus parameters, we used the following parameters torsional Amplitude was 25 linear, phaco power 
was 0 , vaccum was 330 linear, irrigation bottle height was $95 \mathrm{~cm} \mathrm{H}_{2} \mathrm{O}$, aspiration flow rate was 24 linear, dynamic Rise was 0 and intelligent-phaco was off. Regarding the cortex parameters: vaccum was 380 and aspiration flow rate was 30 linear. We performed the stop and chop technique for phacoemulsification in all study eyes. Then irrigation aspiration of the cortical matter was performed. Injection of methyl cellulose in the capsular bag and $\mathrm{AC}$ was performed just before implantation of Hydrophilic aspheric monovision silicon foldable single-piece intraocular lens (UFold,

\subsection{Outcomes}

All study patients were subjected to complete ophthalmic examination and underwent preoperative and postoperative measurements of macular volume; foveal, parafoveal (including both superior and

\subsection{Sample size}

The study participants were divided into two main groups: the non-diabetic and the diabetic groups. Each group included 32 eyes of 32 patients. All 64

\subsection{Statistical methods}

Data was analyzed using STATA version 14.2 (Stata Statistical Software: Release 14.2 College Station, TX: Stata Corp LP.). Quantitative data was represented as mean, standard deviation, median and range. Data was analyzed using student t-test to compare means of two groups and ANOVA for comparison of the means of three groups or more. When the data was not normally distributed Kruskal Wallis test for comparison of three or more groups and Mann-Whitney test was used to compare two groups. Qualitative data was presented as number

\section{Results}

This study included 64 eyes of 64 patients, one eye from each patient, 33 males $(51.6 \%)$ and 31 females $(48.4 \%)$. In group $A$, the mean age of the patient was $59.59 \pm 5.20$ years while in group B, the mean age was $58.13 \pm 3.88$ years old.
Action Medical Pvt. Ltd, Maharashtra, India) in all the study eyes. Closure of the incisions was performed by its hydration with Ringer's lactate solution. Finally, we instilled topical moxifloxacin hydrochloride $0.5 \%$ eye drops (Vigamox, Alcon laboratories Inc., Forth Worth, Texas, USA) and prednisolone acetate $1 \%$ eye drops (Econopred plus ,Alcon laboratories Inc., Forth Worth, Texas, USA) followed by eye patching. At the end we documented the cumulative dissipated energy (CDE) used in each case.

inferior hemisphere) and perifoveal (including both superior and inferior hemisphere) thicknesses) at postoperative months 1, 3 and 6.

eyes were subjected to phacoemulsification with IOL implantation under local anesthetic agents.

and percentage and compared using either Chi square test or fisher exact test. Comparison was made between preoperative and postoperative follow up data at 1 , 3 and 6 months using RMANOVA test. Sphericity were examined using Mauchly's Test of Sphericity. Bonferroni post hoc test was used to examine the difference at each time point. The different time points used as within subject factors. Graphs were produced by either STATA or Excel program. $\mathrm{P}$ value was considered significant if it was less than 0.05 .

We documented no statistically significant differences of the preoperative baseline values between the non-diabetics and diabetic groups, tab. (1). Table (2) summarized the comparative analysis between the non-diabetic and diabetic groups 
regarding the macular OCT outcomes. We recorded statistically significant differences between both groups in the preoperative and postoperative mean outcomes of all macular OCT parameters except mean perifoveal, perifoveal superior and inferior hemispheres $(\mathrm{P}=0.14,0.08$ and 0.99 respectively. The table is also, summarized the differences between the non-diabetic and diabetic groups at all study time-points. Surprisingly, the nondiabetic patients had a higher preoperative mean macular volume than the diabetic patients $(\mathrm{P}=0.01)$, tab. (2). These preoperative significant differences between both groups could be explained by the greater preoperative mean AL in the nondiabetic than the diabetic groups $(\mathrm{P}=$ 0.01 ), tab. (3). However, this preoperative differences did not affect the postoperative outcomes because the detailed analysis of each group alone throughout the entire study time-points revealed that this significant increase in the mean macular volume in the non-diabetic group showed rapid recovery after postoperative month 1 in contrast to the diabetic group which showed slow and incomplete recovery after postoperative month 3 as explained before. Finally, both groups showed insignificant postoperative differences (postoperative month 6 - preoperative outcome) at postoperative month $6(\mathrm{P}=0.4)$, tab. (2) which confirmed that the preoperative significant differences values, tab. (2) had no role in the both groups' subsequent response to surgical procedure regarding these macular changes. Another factor that reinforced this issue was that in the diabetic group we used lower the mean CDE and total US-time values compared to that used in the non-diabetic group, tab. (4).

Complications: Our study recorded no intraoperative complications; however, Table 5 summarized the postoperative complications. We documented four cases of the entire 64 eyes (6\%) complicated with PPME in groups A and $\mathrm{B}$ at the postoperative month 1. Both A and $B$ groups exhibited the same incidence of postoperative macular edema i.e. two (6\%) cases in each group.

Table 1: Patient characteristics

\begin{tabular}{|c|c|c|c|}
\hline Variable & $\begin{array}{c}\text { Group A }(\mathrm{N}=32 \text { eyes of } 32) \\
\text { non-diabetic patients }\end{array}$ & $\begin{array}{c}\text { Group B (N=32 eyes of } 32) \\
\text { diabetic patients }\end{array}$ & $P$ value \\
\hline $\begin{array}{l}\text { Age/years } \\
\text { - Mean } \pm \text { SD } \\
\text { - Median (range) } \\
\end{array}$ & $\begin{array}{c}59.59 \pm 5.20 \\
60(50: 67) \\
\end{array}$ & $\begin{array}{c}58.13 \pm 3.88 \\
57(52: 65) \\
\end{array}$ & 0.21 \\
\hline $\begin{array}{l}\text { Gender } \\
\text { - Total Patients (64) } \\
\text { - Males (33) } \\
\text { - Females (31) } \\
\end{array}$ & $\begin{array}{c}32 \\
16(50.00 \%) \\
16(50.00 \%) \\
\end{array}$ & $\begin{array}{c}32 \\
17(53.13 \%) \\
15(46.88 \%) \\
\end{array}$ & 0.80 \\
\hline $\begin{array}{l}\text { Study subgroups } \\
\text { - Eyes (64) } \\
\text { - NI (37) } \\
\text { - NII (27) } \\
\end{array}$ & $\begin{array}{c}32 \\
18(56.25 \%, \mathrm{~A}-\mathrm{NI}) \\
14(43.75 \%, \mathrm{~A}-\mathrm{NII}) \\
\end{array}$ & $\begin{array}{c}32 \\
19(59.38 \%, \mathrm{~B}-\mathrm{NI}) \\
13(40.63 \%, \mathrm{~B}-\mathrm{NII}) \\
\end{array}$ & 0.80 \\
\hline
\end{tabular}

$\mathbf{N I}=$ nuclear senile cataract grade NI hardness, $\mathbf{N I I}=$ nuclear senile cataract grade NII hardness, $\mathbf{S D}=$ standard deviation

Table 2: Comparative analysis of the macular OCT outcomes in group A versus group B.

\begin{tabular}{|c|c|c|c|}
\hline Variable & $\begin{array}{c}\text { Group A / Mean } \pm \text { SD } \\
\text { Median (range) }\end{array}$ & $\begin{array}{c}\text { Group B / Mean } \pm \text { SD } \\
\text { Median (range) }\end{array}$ & $P$ value \\
\hline $\begin{array}{l}\text { Macular volume }\left(\mathbf{m m}^{2}\right) \\
\text { - Preoperative }\end{array}$ & $\begin{array}{c}6.94 \pm 0.47 \\
7.00(5.8: 7.53)\end{array}$ & $\begin{array}{c}6.67 \pm 0.36 \\
6.66(6.14: 7.71)\end{array}$ & 0.01 \\
\hline - Post 1m & $\begin{array}{c}7.07 \pm 0.48 \\
7.09(6: 7.8)\end{array}$ & $\begin{array}{c}6.80 \pm 0.53 \\
6.73(6.08: 8.58)\end{array}$ & 0.03 \\
\hline
\end{tabular}




\begin{tabular}{|c|c|c|c|}
\hline - Post $3 \mathrm{~m}$ & $\begin{array}{c}7.15 \pm 0.60 \\
7.14(6.07: 9.37)\end{array}$ & $\begin{array}{c}6.78 \pm 0.53 \\
6.71(6: 8.57)\end{array}$ & 0.01 \\
\hline - Post $6 \mathrm{~m}$ & $\begin{array}{c}7.05 \pm 0.45 \\
7.16(5.98: 7.8)\end{array}$ & $\begin{array}{c}6.73 \pm 0.47 \\
6.65(6: 8.56)\end{array}$ & 0.001 \\
\hline - Post 6ms-Preoperative & $\begin{array}{c}0.11 \pm 0.24 \\
0.07(-0.26: 0.68)\end{array}$ & $\begin{array}{c}0.06 \pm 0.17 \\
0.04(-0.14: 0.85)\end{array}$ & 0.40 \\
\hline $\begin{array}{l}\text { Foveal thickness }(\mu \mathrm{m}) \\
\text { - Preoperative } \\
\text { - Post } 1 \mathrm{~m}\end{array}$ & $\begin{array}{c}256.84 \pm 21.04 \\
260.5(207: 287) \\
259.06 \pm 24.15 \\
264(204: 299)\end{array}$ & $\begin{array}{l}230.31 \pm 18.66 \\
223(206: 272) \\
234.19 \pm 28.72 \\
222(210: 324)\end{array}$ & $\begin{array}{l}<0.0001 \\
0.0004\end{array}$ \\
\hline - Post $3 \mathrm{~m}$ & $\begin{array}{c}267.16 \pm 52.76 \\
264.5(202: 523)\end{array}$ & $\begin{array}{c}240.35 \pm 34.97 \\
225.5(205: 323)\end{array}$ & 0.02 \\
\hline - Post $6 \mathrm{~m}$ & $\begin{array}{c}258.5 \pm 24.13 \\
262(204: 302)\end{array}$ & $\begin{array}{l}238.69 \pm 33.43 \\
231(205: 323)\end{array}$ & 0.01 \\
\hline - Post 6ms-Preoperative & $\begin{array}{c}1.66 \pm 9.37 \\
-0.5(-18: 21)\end{array}$ & $\begin{array}{c}8.38 \pm 26.78 \\
0.5(-11: 101)\end{array}$ & 0.75 \\
\hline $\begin{array}{l}\text { Parafoveal thickness }(\mu \mathrm{m}) \\
\text { - Preoperative }\end{array}$ & $\begin{array}{l}313.88 \pm 16.89 \\
315(277: 340)\end{array}$ & $\begin{array}{c}294.13 \pm 17.06 \\
292.5(270: 347)\end{array}$ & $<0.0001$ \\
\hline - Post $1 \mathrm{~m}$ & $\begin{array}{c}317.5 \pm 21.09 \\
320.5(276: 352)\end{array}$ & $\begin{array}{c}299.34 \pm 26.04 \\
295.5(276: 395)\end{array}$ & 0.003 \\
\hline - Post $3 \mathrm{~m}$ & $\begin{array}{l}320.19 \pm 27.47 \\
322(278: 419)\end{array}$ & $\begin{array}{l}299.56 \pm 25.94 \\
296(281: 395)\end{array}$ & 0.003 \\
\hline - Post $6 \mathrm{~m}$ & $\begin{array}{c}316.5 \pm 20.90 \\
323(275: 355)\end{array}$ & $\begin{array}{l}297.91 \pm 22.41 \\
294(276: 395)\end{array}$ & 0.001 \\
\hline - Post 6ms-Preoperative & $\begin{array}{c}2.63 \pm 9.58 \\
0.5(-19: 25)\end{array}$ & $\begin{array}{c}3.78 \pm 10.53 \\
3(-19: 48)\end{array}$ & 0.69 \\
\hline $\begin{array}{l}\text { Parafoveal superior hemi- } \\
\text { sphere }(\mu \mathrm{m}) \\
\text { - Preoperative }\end{array}$ & $\begin{array}{c}313.66 \pm 15.81 \\
312.5(283: 338)\end{array}$ & $\begin{array}{c}293.75 \pm 17.45 \\
292.5(268: 349)\end{array}$ & $<0.0001$ \\
\hline - Post $1 \mathrm{~m}$ & $\begin{array}{c}319 \pm 19.53 \\
321(283: 350)\end{array}$ & $\begin{array}{c}298.5 \pm 27.52 \\
293(275: 399)\end{array}$ & 0.001 \\
\hline - Post $3 \mathrm{~m}$ & $\begin{array}{c}322 \pm 27.64 \\
319.5(280: 427)\end{array}$ & $\begin{array}{l}298.94 \pm 27.19 \\
294(280: 398)\end{array}$ & 0.001 \\
\hline - Post $6 \mathrm{~m}$ & $\begin{array}{c}318.28 \pm 20.29 \\
320.5(282: 362)\end{array}$ & $\begin{array}{c}297.22 \pm 23.42 \\
297.5(274: 398)\end{array}$ & 0.0003 \\
\hline - Post 6ms -Preoperative & $\begin{array}{c}4.63 \pm 10.41 \\
3(-18: 30)\end{array}$ & $\begin{array}{c}3.47 \pm 12.14 \\
3(-18: 49)\end{array}$ & 0.61 \\
\hline $\begin{array}{l}\text { Parafoveal inferior hemi- } \\
\text { sphere }(\mu \mathrm{m}) \\
\text { - Preoperative }\end{array}$ & $\begin{array}{l}313.91 \pm 18.97 \\
320(260: 341)\end{array}$ & $\begin{array}{c}295.13 \pm 16.56 \\
293.5(270: 345)\end{array}$ & 0.0001 \\
\hline
\end{tabular}




\begin{tabular}{|c|c|c|c|}
\hline - Post 1m & $\begin{array}{c}315.88 \pm 23.25 \\
321.5(259: 354)\end{array}$ & $\begin{array}{l}300.38 \pm 24.78 \\
296(276: 391)\end{array}$ & 0.01 \\
\hline - Post 3m & $\begin{array}{c}318.34 \pm 27.94 \\
323.5(260: 411)\end{array}$ & $\begin{array}{c}300.25 \pm 24.87 \\
296.5(282: 392)\end{array}$ & 0.008 \\
\hline - Post 6m & $\begin{array}{c}314.78 \pm 22.24 \\
322.5(259: 351)\end{array}$ & $\begin{array}{c}298.72 \pm 21.56 \\
294.5(278: 392)\end{array}$ & 0.005 \\
\hline - Post 6ms-Preoperative & $\begin{array}{c}0.88 \pm 9.75 \\
0.5(-19: 22)\end{array}$ & $\begin{array}{c}3.59 \pm 10.28 \\
3(-9: 47)\end{array}$ & 0.27 \\
\hline Perifoveal $(\mu \mathrm{m})$ & & & \\
\hline - Preoperative & $\begin{array}{l}272.63 \pm 23.79 \\
278(215: 302)\end{array}$ & $\begin{array}{c}267.38 \pm 15.55 \\
266.5(242: 308)\end{array}$ & 0.30 \\
\hline - Post 1m & $\begin{array}{l}277.13 \pm 23.15 \\
281(216: 309)\end{array}$ & $\begin{array}{l}270.88 \pm 23.52 \\
268(223: 341)\end{array}$ & 0.29 \\
\hline - Post 3m & $\begin{array}{l}278.03 \pm 23.52 \\
282(216: 309)\end{array}$ & $\begin{array}{c}271.13 \pm 23.73 \\
266.5(220: 340)\end{array}$ & 0.25 \\
\hline - Post 6m & $\begin{array}{l}276.72 \pm 23.23 \\
281(213: 308)\end{array}$ & $\begin{array}{l}268.34 \pm 21.85 \\
263(220: 340)\end{array}$ & 0.14 \\
\hline - Post 6ms-Preoperative & $\begin{array}{l}4.09 \pm 11.37 \\
0.5(-10: 42)\end{array}$ & $\begin{array}{c}0.97 \pm 9.12 \\
1.0(-22: 32)\end{array}$ & 0.69 \\
\hline $\begin{array}{l}\text { Perifoveal superior hemi- } \\
\text { sphere }(\mu \mathrm{m}) \\
\text { - Preoperative }\end{array}$ & $\begin{array}{c}273.16 \pm 25.40 \\
280.5(191: 299)\end{array}$ & $\begin{array}{l}269.06 \pm 16.17 \\
269(237: 310)\end{array}$ & 0.44 \\
\hline - Post $1 \mathrm{~m}$ & $\begin{array}{c}279.25 \pm 22.39 \\
283.5(209: 309)\end{array}$ & $\begin{array}{c}270.94 \pm 26.11 \\
269.5(206: 341)\end{array}$ & 0.18 \\
\hline - Post 3m & $\begin{array}{c}280.5 \pm 22.23 \\
283.5(210: 309)\end{array}$ & $\begin{array}{l}272.38 \pm 26.19 \\
269(205: 342)\end{array}$ & 0.19 \\
\hline - Post 6m & $\begin{array}{l}279.41 \pm 22.59 \\
\quad(207: 310)\end{array}$ & $\begin{array}{c}268.81 \pm 24.52 \\
265.5(205: 342)\end{array}$ & 0.08 \\
\hline - Post 6ms-Preoperative & $\begin{array}{c}06.25 \pm 14.74 \\
1.5(-13: 63)\end{array}$ & $\begin{array}{c}-0.25 \pm 11.10 \\
2(-32: 32)\end{array}$ & 0.14 \\
\hline $\begin{array}{l}\text { Perifoveal inferior hemisp- } \\
\text { here }(\boldsymbol{\mu m}) \\
\text { - Preoperative }\end{array}$ & $\begin{array}{c}272 \pm 25.43 \\
277.5(186: 304)\end{array}$ & $\begin{array}{c}266 \pm 15.26 \\
262(244: 305)\end{array}$ & 0.26 \\
\hline - Post $1 \mathrm{~m}$ & $\begin{array}{l}274.97 \pm 25.24 \\
278(204: 308)\end{array}$ & $\begin{array}{c}270.88 \pm 22.08 \\
268.5(240: 341)\end{array}$ & 0.49 \\
\hline - Post 3m & $\begin{array}{c}275.63 \pm 26.42 \\
279.5(190: 311)\end{array}$ & $\begin{array}{c}276.13 \pm 25.37 \\
266.5(250: 338)\end{array}$ & 0.94 \\
\hline - Post 6m & $\begin{array}{l}274.03 \pm 25.75 \\
278(191: 307)\end{array}$ & $\begin{array}{c}274.13 \pm 24.35 \\
263.5(248: 338)\end{array}$ & 0.99 \\
\hline - Post 6ms-Preoperative & $\begin{array}{c}2.03 \pm 10.4 \\
1.5(-16: 39) \\
\end{array}$ & $\begin{array}{c}8.13 \pm 22.38 \\
3(-12: 88) \\
\end{array}$ & 0.19 \\
\hline
\end{tabular}

$P$ values were calculated using either Student's t-test for normally distributed data or Mann-Whitney test for non-normally distrusted data 
Table 3: Comparison between groups A versus B as regards some preoperative data

\begin{tabular}{|l|c|c|c|}
\multicolumn{1}{|c}{ Variable } & $\begin{array}{c}\text { Group A / Mean } \pm \text { SD } \\
\text { Median (range) }\end{array}$ & $\begin{array}{c}\text { Group B / Mean } \pm \text { SD } \\
\text { Median (range) }\end{array}$ & \multicolumn{2}{c|}{ P value } \\
\hline Pre-operative axial lens $(\mathbf{m m})$ & $23.80 \pm 1.60$ & $22.91 \pm 1.08$ & $\mathbf{0 . 0 1}$ \\
& $23.54(21.21: 28.01)$ & $22.97(21.56: 25.22)$ & \\
Anterior chamber Depth $(\mathbf{m m})$ & $3.27 \pm 0.33$ & $3.22 \pm 0.37$ & $\mathbf{0 . 5 9}$ \\
& $3.28(2.62: 3.96)$ & $3.19(2.57: 3.88)$ & \\
\hline
\end{tabular}

Table 4: Comparative analysis of the intraoperative outcomes in group A versus group B

\begin{tabular}{|l|c|c|c|}
\hline \multicolumn{1}{|c}{ Variable } & $\begin{array}{c}\text { Group A / Mean } \pm \text { SD } \\
\text { Median (range) }\end{array}$ & \multicolumn{1}{c|}{$\begin{array}{c}\text { Group B / Mean } \pm \text { SD } \\
\text { Median (range) }\end{array}$} & \multicolumn{1}{c|}{ P value } \\
\hline Cumulative Dissipated Energy & $5.37 \pm 3.54$ & $4.68 \pm 2.99$ & $\mathbf{0 . 6 6}$ \\
US total (torsional) time(seconds) & $4.18(0.86: 16.85)$ & $4.66(0.76: 9.86)$ & \\
& $27.79 \pm 16.72$ & $25.43 \pm 12.81$ & $\mathbf{0 . 9 5}$ \\
Estimated fluid used(CC) & $22.25(2.7: 75.7)$ & $27.35(7.2: 45.7)$ & \\
& $96.44 \pm 27.44$ & $110.69 \pm 33.82$ & $\mathbf{0 . 1 5}$ \\
\hline
\end{tabular}

Table 5: Postoperative complications in the two study groups

\begin{tabular}{|c|c|c|c|c|}
\hline Complications & $\begin{array}{c}\text { Group A } \\
\mathbf{N}=32\end{array}$ & $\begin{array}{c}\text { Group B } \\
\mathbf{N}=32\end{array}$ & Management & Fate \\
\hline PPME & $\begin{array}{l}2 \\
(6 \%)\end{array}$ & $\begin{array}{l}2 \\
(6 \%)\end{array}$ & $\begin{array}{l}\text { - Conservative management } \\
\text { - Topical steroid eye drops in the } \\
\text { form of prednisolone acetate } 1 \% \\
\text { (Pred forte, Allergan, inc, jersey } \\
\text { city, USA) } \\
\text { - Topical non-steroidal anti- } \\
\text { inflammatory dugs (Nevanac, } \\
\text { Alcon Laboratories, inc) in adose } \\
\text { of one drop three times daily } \\
\text { - Systemic non-steroidal anti- } \\
\text { inflammatory dugs Ibuprofen } \\
\text { 400 mg tablets (Brufen } 400 \mathrm{mg} \text {, } \\
\text { Abbot pharmaceuticals, Illinios, } \\
\text { USA). } \\
\text { - Acetazolamide tablet } 250 \text { mg } \\
\text { twice per day (Cidamex }\end{array}$ & $\begin{array}{l}\text { - PPME in the } 2 \text { eyes } \\
\text { of group A showed } \\
\text { rapid and complete } \\
\text { recovery within 2-4 } \\
\text { weeks with no subs- } \\
\text { equent sequelae } \\
\text { - PPME in the } 2 \text { eyes } \\
\text { of group B showed } \\
\text { gradual and in compl- } \\
\text { ete recovery till end of } \\
\text { the study no improvem- } \\
\text { ent in the final CDVA }\end{array}$ \\
\hline
\end{tabular}

$\boldsymbol{P C O}=$ posterior capsular opacification, $\boldsymbol{P P M E}=$ post-phacoemulsification macular edema .

Figures (1-4) shows an example of PPME in 55 years old male patient in the non-diabetic group with a cataract density NII, preoperative CDVA was $1 \log$ MAR, during surgery CDE used was 5.50. The postoperative CDVA was $0 \log$ MAR at first postoperative week. However, at end of postoperative month 1, PPME developed and revealed more severity within one week as the macula became domeshaped while CDVA deteriorated to 0.50 $\log$ MAR. Finally, PPME resolved rapidly and completely within 2 weeks with improvement CDVA to 0 logMAR again. Meanwhile, fig. . (5-7) shows an example of PPME in 55 years old female patient in the diabetic group with a cataract density NII, preoperative CDVA was 0.8 $\operatorname{logMAR}$, during surgery CDE used was 6.07. The postoperative CDVA was 0.2 $\log$ MAR at first postoperative week. However, at end of postoperative month 1, PPME developed and while CDVA deteriorated to $0.50 \log M A R$. Finally, PPME resolved gradually and incompletely but, unfortunately, did not resolve totally till the end of the study with no improvement in the patient CDVA that remained stable at $0.3 \log$ MAR. 


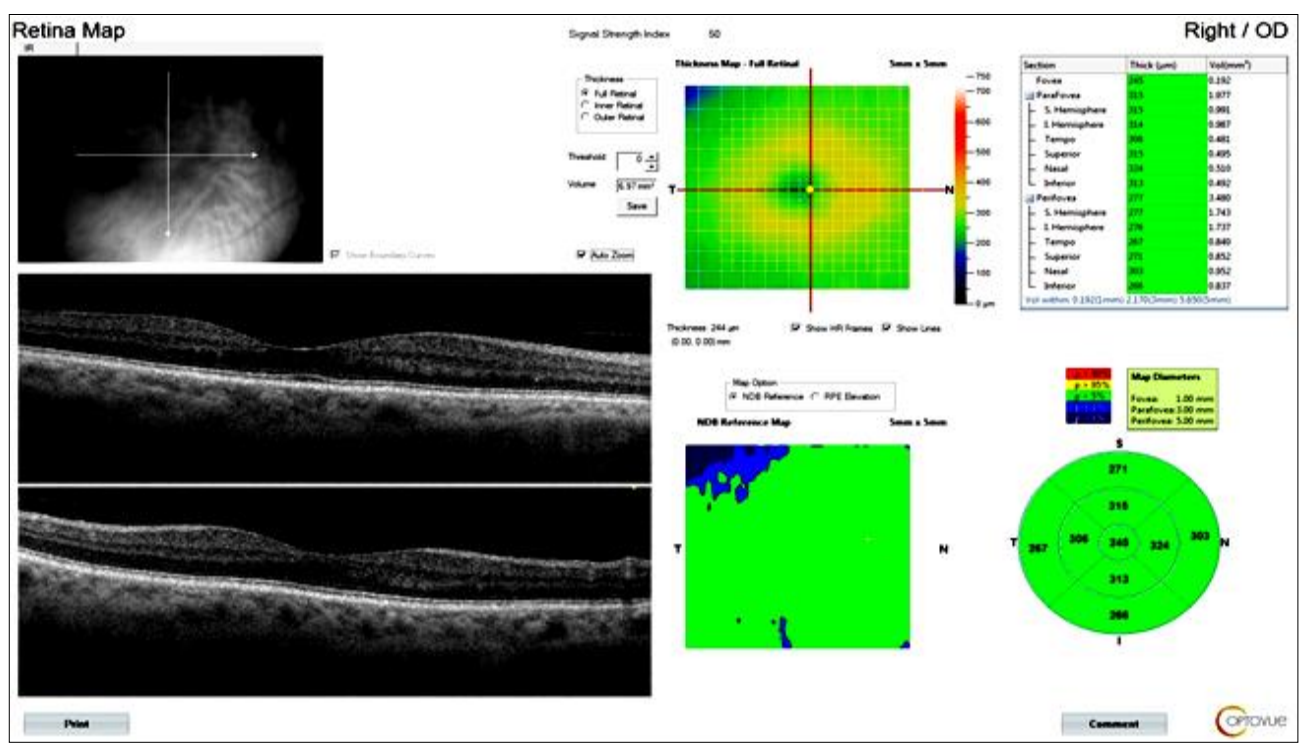

Figure 1. Preoperative macular map of the patient in group A

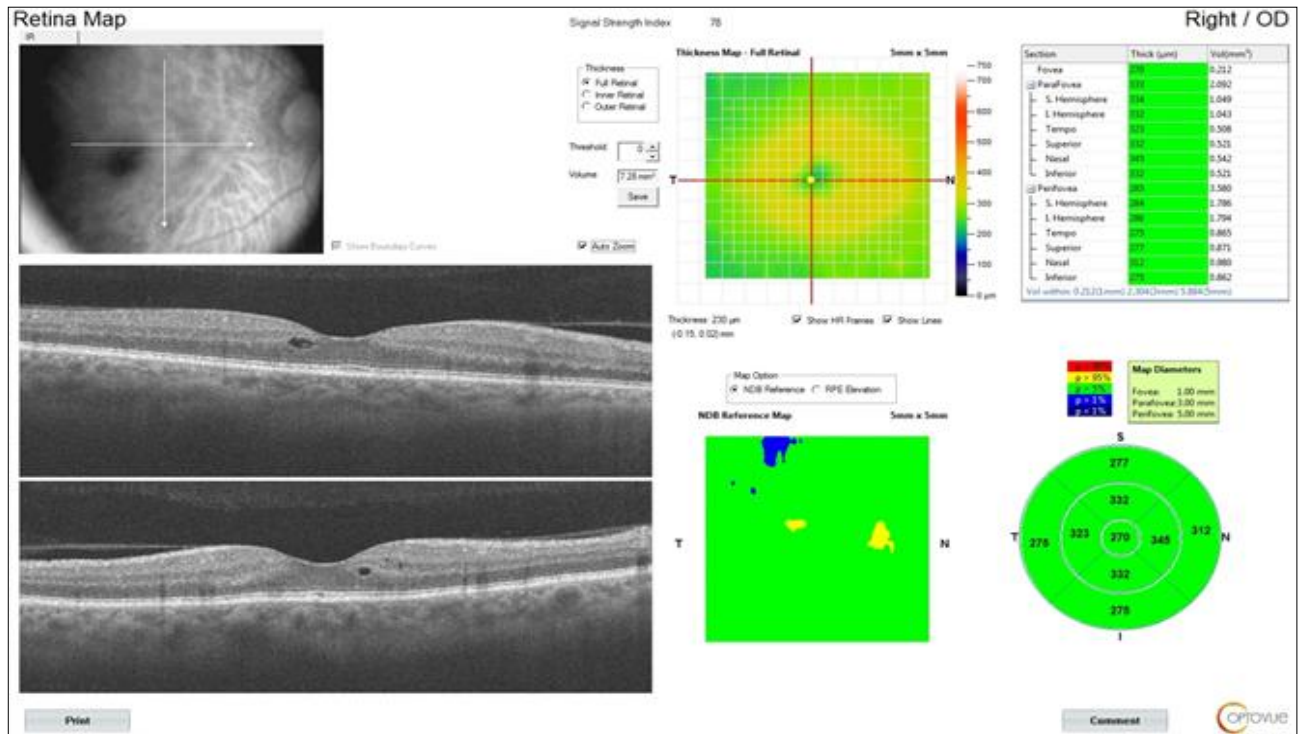

Figure 2. Macular map 23 day postoperative of the patient in group A

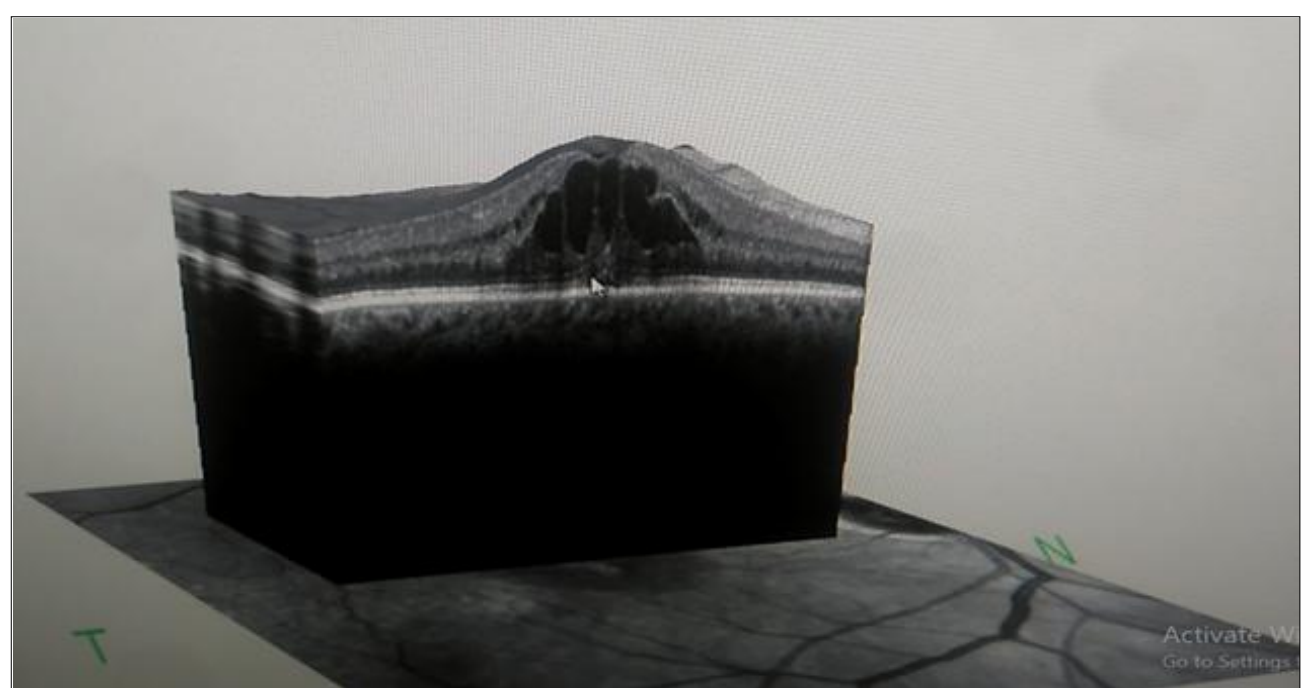

Figure 3 . Radial view 36 days postoperative of the patient in group A 


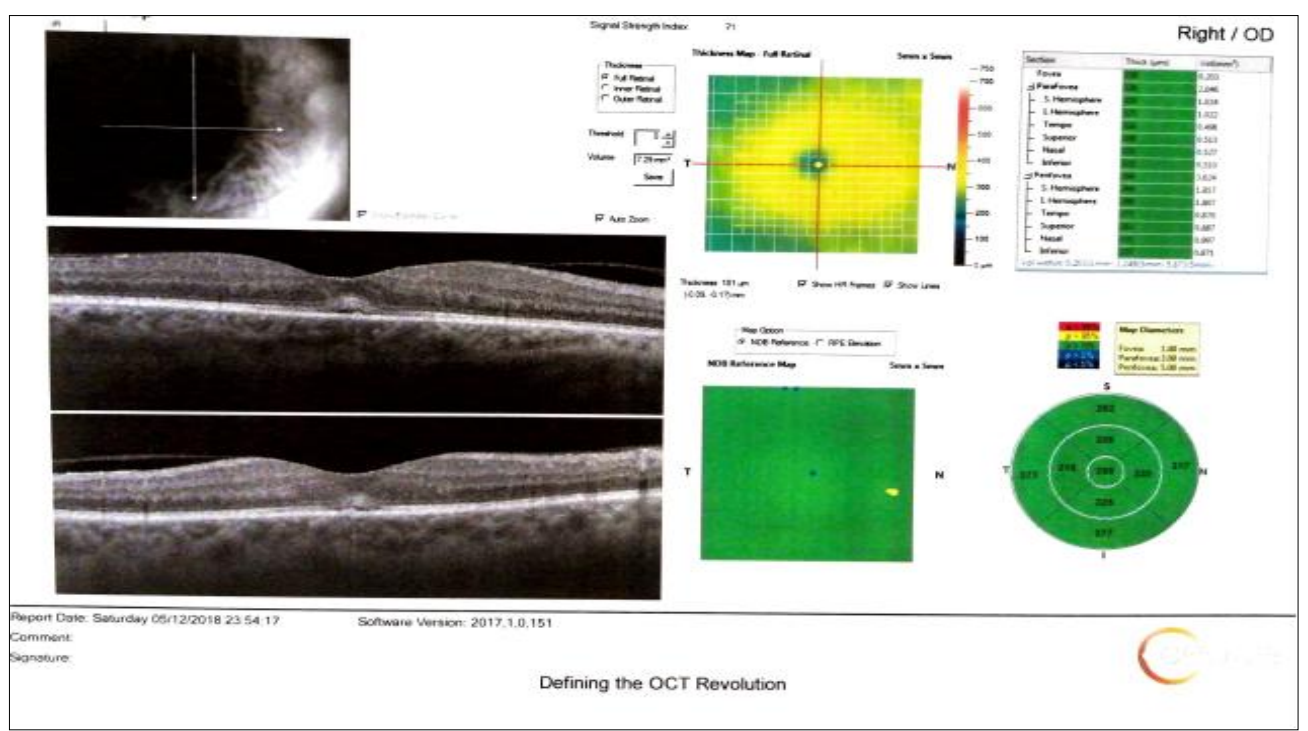

Figure 4. Retinal map 43 days postoperative of the patient in group A

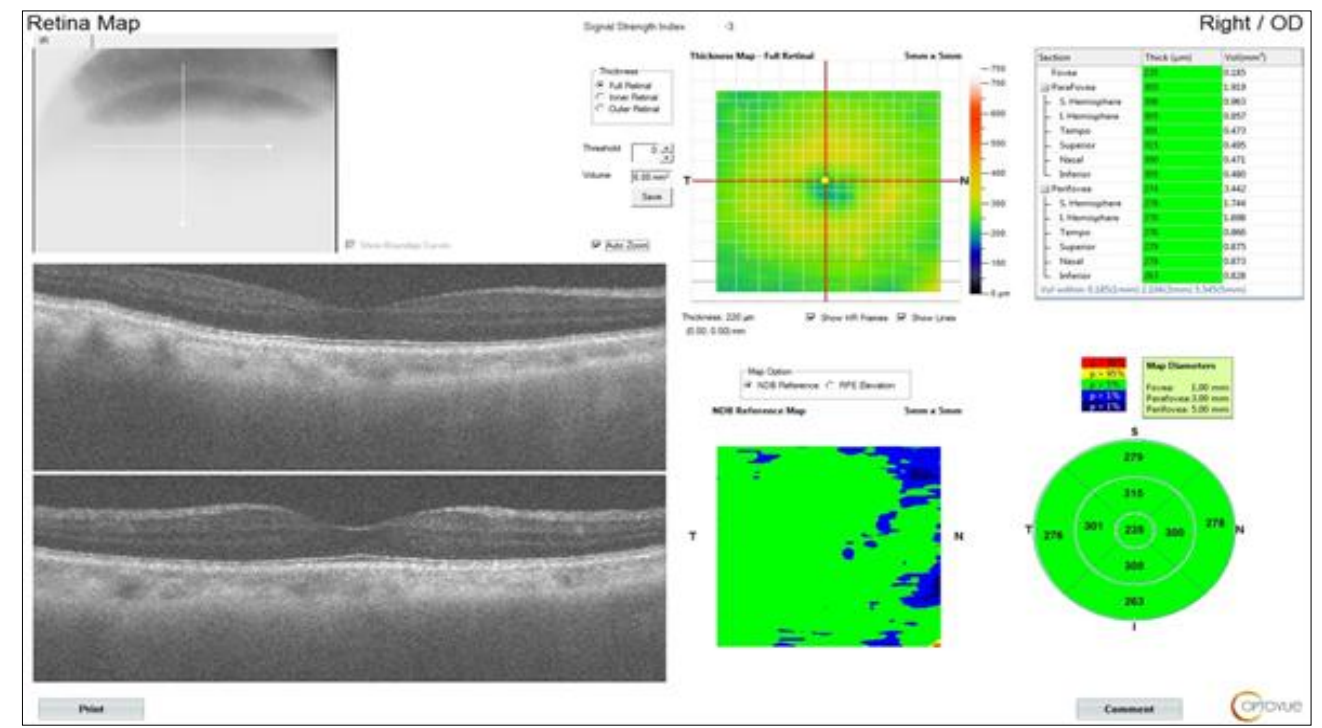

Figure 5. Preoperative macular map of the patient in group B

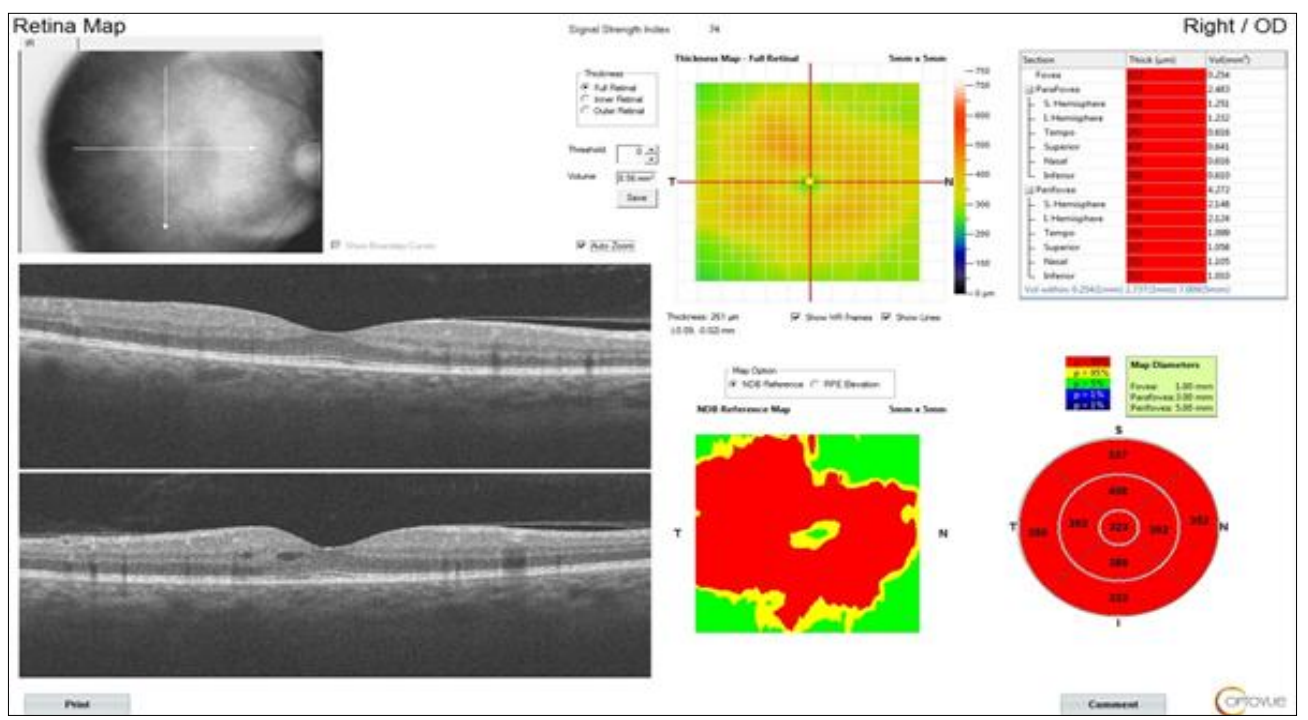

Figure 6. Macular map 30 days postoperative of the patient in group B 


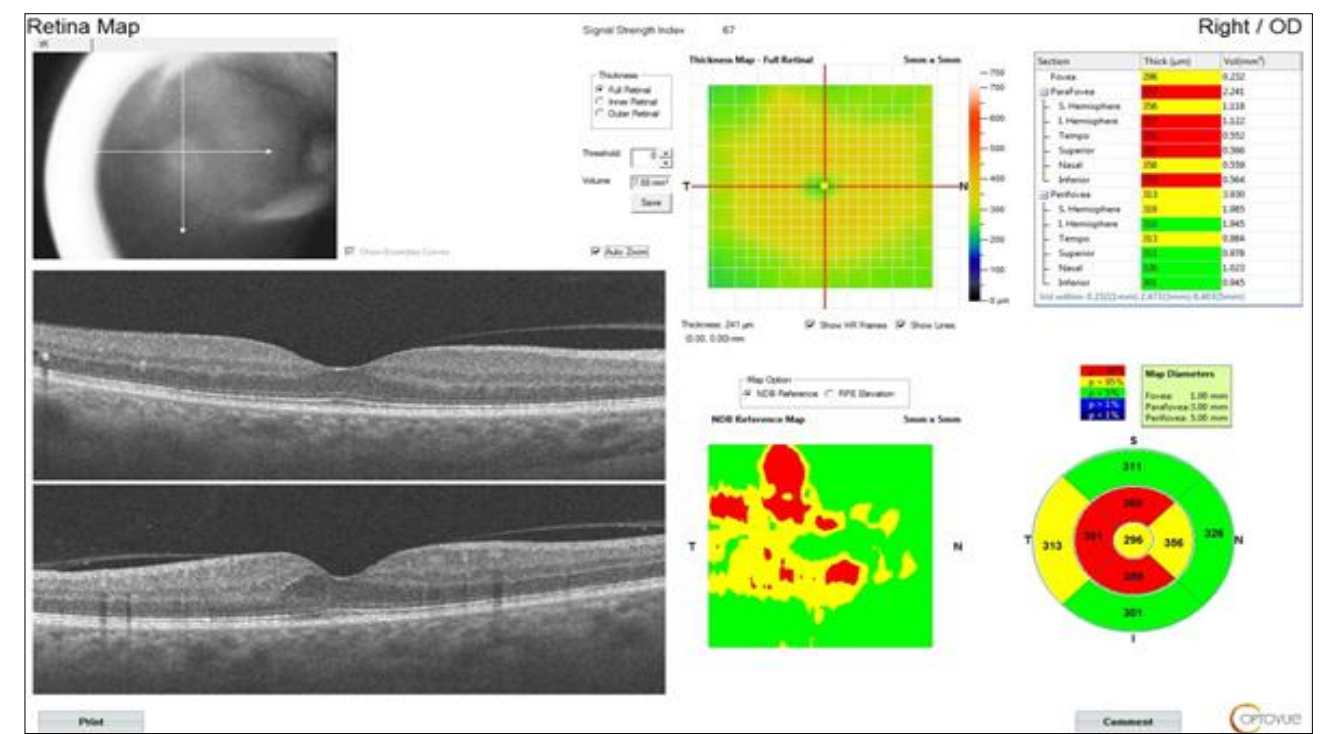

Figure 7. Macular map 6 month postoperative of the patient in group B at the end of follow up

\section{Discussion}

This study included 64 eyes of 64 patients (32 non-diabetic and 32 diabetic patients) that underwent phacoemulsification and posterior chamber IOL implantation for treatment of the visually significant nuclear senile cataract (nucleus type NI and NII). Regarding the developed PPME, our study documented that there was equal incidence of PPME development in both non-diabetic and diabetic groups (2 cases in each group). This finding was questioned by our team; however, we suggested that the PPME development was directly related to the surgical procedure itself and was not directly related to being a diabetic or non-diabetic patient in absence of diabetic retinopathy. Furthermore, we also documented that the two cases in the non-diabetic group recovered rapidly and PPME resolved totally within 2-4 weeks following its diagnosis. On the other hand, the other two cases of PPME in the diabetic group, unfortunately, revealed slow recovery with gradual improvement but not totally resolved till the end of the study. Therefore, we believe that there is an equal chance of postoperative development of PPME in the non-retinopathy diabetic versus non-diabetic patients. We simply could explain our belief on the basis that this PPME development is directly related to the surgical procedure itself and not due to diabetes mellitus affection. Finally, we also believe that the recovery from the PPME is directly related to the presence or absence of DM and not related to surgical procedure itself. On the other hand, Kwon et al., retrospectively analyzed the incidence of PPME in non-retinopathy and retinopathy diabetic patients. In their the non-retinopathy diabetics, they recorded a high incidence of PPME (20\%) that peaked at postoperative month 1 that revealed spontaneous resolution in two thirds of cases at postoperative month 6 [10]. In comparison to our study, we only documented much lower incidence $(6 \%)$ of PPME in nonretinopathy diabetics. In their database retrospective study, Chu et al reported an incidence of $1.17 \%, 2.15 \%$ and $7.27 \%$ regarding PPME in the non-diabetics, nonretinopathy diabetics and the diabetics with retinopathy, respectively [11]. In contrast to their findings, we documented $6 \%$ incidence of PPME in both nondiabetics and non-retinopathy diabetics. However, this great difference between both studies was their huge sample size (81984 eyes) in contrast to our small 
sample size (64 eyes). Wang et al., explained the general tendency of macular and retinal thickening in diabetics following phacoemulsification by the postoperative reduction in the parafoveal vessel density in the deep capillary plexus as evidenced by the postoperative macular OCT changes [12]. Furthermore, such changes were absent in the non-diabetics and in the superficial capillary plexus. The main differences between both studies that we documented equal 6\% PPME in both groups, however, both studies agreed that
PPME was directly related to CDE of Phaco-machine. Ikegami et al recorded the macular, choroidal and aqueous flare changes following small-incision cataract surgery. They finally concluded that there was an increased inflammation induced by cataract surgery ending in both postoperative macular and choroidal thickening with increased aqueous flare in the diabetic group. The main differences between their study and ours was the type of cataract surgery [13].

\section{Conclusions}

Our outcomes confirmed that there was a similar probability of PPME occurrence in both nondiabetic and diabetic patients as this probability was mostly related to the surgical procedure itself. Meanwhile, the non-diabetic PPME exhibited rapid and complete recovery within one month in comparison to the diabetic PPME that exhibited gradual and incomplete recovery during the postoperative six months follow-up period. Therefore, we think that both groups had no differences regarding PPME development, yet the differences between both groups could be attributed to the good recovery process in short time in the non-diabetic patients. Finally, we simply believe that the extent of the cell injury, loss and subsequent edema is directly related to the intraoperative surgical insult regardless being a diabetic or non-diabetic patient. However, we also believe that the postoperative ability of the cells to heal, repair and restore its functions depends on the health of these cells that is directly related to being a diabetic or non-diabetic patient

\section{References}

1. Memon A., Mahar P., Memon M., et al. Age-related cataract and its types in patients with and without type 2 diabetes mellitus: A Hospital-based comparative study. J Pak Med Assoc. 2016; 66 (10): 1272-1276.

2. Abib FC. Dos Santos RM Corneal Endothelium: Histology, Physiology and In-vivo Examination with Specular Microscope. JSM Ophthalmol. 2017; 5 (4): $1063----$.

3. Praveen M., Vasavada A., Shah G., et al. A prospective evaluation of posterior capsule opacification in eyes with diabetes mellitus: a case-control study. Eye (Lond). 2014; 28(6): 720-727.

4. Morikubo S., Takamura Y., Kubo E., et al. Corneal changes after small-incision cataract surgery in patients with diabetes mellitus. Arch Ophthalmol. 2004; 122 (7): 966-969.
5. Sies H. Oxidative stress: Oxidants and antioxidants. Exp Physiol. 1997; 82 (2): 291-295.

6. Halliwell B. Free radicals and antioxidants: Updating a personal view. Nutr Rev. 2012; 70 (5): 257-265.

7. Shah A. \& Chen S. Cataract surgery and diabetes. Curr Opin Ophthalmol. 2010; 21 (1): 4-9.

8. Ursell P., Spalton D., Whitcup S., et al. Cystoid macular edema after phacoemulsification: relationship to bloodaqueous barrier damage and visual acuity. J Cataract Refract Surg. 1999; 25(11):1492-1497.

9. Chylack Jr., Wolfe J., Singer D., et al. The Lens Opacities Classification System III. The Longitudinal Study of Cataract Study Group. Arch Ophthalmol. 1993; 111 (6): 831-836.

10. Kwon S., Hwang D., Seo J., et al Evaluation of changes of macular 
thickness in diabetic retinopathy after cataract surgery. Korean J Ophthalmol. 2011; 25 (4): 238-242.

11. Chu C., Johnston R., Buscombe C., et al. Risk factors and incidence of macular edema after cataract surgery: A database study of 81984 eyes. Ophthalmology. 2016; 123 (2): 316323.

12. Wang Z., Wang E., Chen Y. Transient reduction in macular deep capillary density on optical coherence tomogr- aphy angiography after phacoemulsification surgery in diabetic patients. BMC Ophthalmol. 2020; 20 (1): 335---.

13. Ikegami Y. Takahashi M., Amino K. Evaluation of choroidal thickness, macular thickness, and aqueous flare after cataract surgery in patients with and without diabetes: A prospective randomized study. BMC Ophthalmol. 2020; 20 (1): 102----. 